\title{
Motion-Resistant Pulse Oximetry Processing Based on Time-Frequency Analysis
}

\author{
Theera Leeudomwong ${ }^{1, a}$, Tayard Deesudchit ${ }^{2}$, and Chedsada Chinrungrueng ${ }^{3, \mathrm{~b}, *}$ \\ 1 Biomedical Engineering Programme, Chulalongkorn University, Bangkok 10330, Thailand \\ 2 Department of Paediatrics, Faculty of Medicine, Chulalongkorn University, Bangkok 10330, Thailand \\ 3 Department of Electrical Engineering, Faculty of Engineering, Chulalongkorn University, Bangkok \\ 10330, Thailand \\ E-mail: atheera.1@hotmail.com, bchedsada.c@chula.ac.th (Corresponding author)
}

\begin{abstract}
This paper proposes an alternative method for pulse oximetry processing based on time-frequency analysis. Instead of using the fast Fourier transform (FFT), the continuous wavelet transform (CWT) together with the median calculation was proposed for finding the frequency components of the normalized first derivative red and infrared photoplethysmographic signals (PPG). In order to estimate the arterial oxygen saturation $\left(\% \mathrm{SpO}_{2}\right)$, the ratio of each red CW'T modulus to each corresponding infrared CWT modulus was computed at each frequency, and then converted to the oxygen saturation by using the saturation equation obtained from calibration. All of the oxygen saturation data were plotted on a histogram. The greatest oxygen saturation with the most occurrences was considered to represent the $\mathrm{oSpO}_{2}$. The experiments were held to evaluate the performance of the proposed processing method compared with the conventional pulse oximetry $(\mathrm{CPO})$ processing. The red and infrared PPGs were acquired from left and right index fingers simultaneously by using the PPG measurement system constructed in the laboratory. During measurement, the left index finger was stationary, while the right index finger was performed the finger bend to induce the motion artifact (MA) in transient and periodic manners. All detected PPGs were processed by the proposed method and the $\mathrm{CPO}$ processing. The $\% \mathrm{SpO}_{2}$ obtained from different processing methods and positions were compared. Experimental results showed that the proposed method was more resistant to the MA than the conventional method.
\end{abstract}

Keywords: Photoplethysmography, pulse oximetry, motion artifact, time-frequency analysis, continuous wavelet transform.

ENGINEERING JOURNAL Volume 21 Issue 7

Received 15 February 2017

Accepted 29 May 2017

Published 29 December 2017

Online at http:/ /www.engj.org/

DOI:10.4186/ej.2017.21.7.181 


\section{Introduction}

Pulse oximetry, an optical technique for measuring functional oxygen saturation, was invented by Takuo Aoyagi in 1972 [1, 2]. By this invention, the arterial blood oxygenation can be measured conveniently, quickly, inexpensively, continuously, and non-invasively. Conventional pulse oximetry (CPO) relies on detecting the photoplethysmogram (PPG), of which the amplitude varies with the light absorption of the arterial blood and depends on the arterial blood volume and oxygenation [3]. CPO processing generally requires two PPGs, approximately red and infrared (IR), for finding the ratio of the arterial light absorption between red and IR, which theoretically relates to the arterial oxygen saturation. The pulse oximeter, which is an instrument using the pulse oximetry for measuring the arterial oxygen saturation $\left(\% \mathrm{SpO}_{2}\right)$, plays a significant role in providing the patient's oxygen status, and has been extensively used for patient monitoring in critical care units as well as for diagnostic purposes $[4,5]$.

The motion artifact (MA), an undesired signal induced by body movement, is a major problem of the pulse oximeter using CPO processing. It often misinterprets the MA as the PPG and leads to an inaccurate reading, false desaturation alarm, and missed true desaturation alarms [6].

Over the years, there have been a number of attempts to reduce the impact of MA on CPO processing. A primary method was to improve the probe design, which helped reduce and protect against probe displacement and ambient light interference. Most studies focused on the elimination of MA from the corrupted PPG by applying different signal processing techniques; for example, the Fourier series analysis [7], the adaptive filtering [8-12], the independent component analysis [12-15], and the wavelet transform [15, 16]. A major drawback of these techniques is the overlapping of the frequency band between the MA and PPG.

One possible solution was to develop motion resistant algorithms for pulse oximetry processing instead of the CPO. The main concept of this approach is to revise the conventional PPG model by adding a term representing the attenuation of light intensity induced by movement, and to develop a signal processing method for estimating the $\% \mathrm{SpO}_{2}$ from the revised model. Goldman, Petterson, Kopotic, and Barker (2000) and Diab et al. (2009) presented several pulse oximetry technologies developed by the Masimo Corporation. The PPG model was revised in the belief that the MA originated from the variation of light absorption from venous blood and other non-arterial substances as they move. A renowned algorithm is the discrete saturation transform (DST), which is a motion-resistant algorithm implemented by using the adaptive filter [17].

Frequency domain analysis is another interesting approach for pulse oximetry processing. Diab et al. (2009) presented an algorithm based on using the fast Fourier transform (FFT). In this algorithm, the red and IR PPGs are normalized by using the logarithmic method and decomposed into different frequencies by using the FFT. Then, the ratio of each red FFT coefficient to each corresponding infrared FFT coefficient is computed at each frequency. The oxygen saturation relating to each ratio is determined by using the saturation equation. Finally, all of the saturation data are counted on a histogram, and the oxygen saturation with the highest number of occurrences is considered to represent the $\% \mathrm{SpO}_{2}$ [18].

According to the algorithm proposed by Diab et al. (2009) mentioned above, we noticed that the efficiency of this algorithm was limited by the resolution of the frequency spectrum provided by the FFT. The low-frequency resolution results in less saturation data, which may lead to misidentifying the $\% \mathrm{SpO}_{2}$, especially when the detected PPGs are corrupted with the MA. Therefore, this algorithm could be more powerful if the spectral resolution of the frequency spectrum can be increased.

In this work, we focused on the development of motion-resistant pulse oximetry processing. We propose an alternative signal processing method based on the time-frequency analysis that improves the algorithm proposed by Diab et al. (2009). Instead of the frequency analysis using the FFT, we suggest applying timefrequency (TF) analysis together with the median calculation in order to increase the spectral resolution of the frequency spectrum of the normalized PPG, resulting in increasing the number of saturation data to be plotted onto a histogram. Therefore, the $\% \mathrm{SpO}_{2}$ is revealed more clearly, and can be identified more easily, particularly when the PPGs are contaminated with the MA. Among the many TF techniques available, such as the short time Fourier transform (STFT), Wigner-Ville transform (WVT), Choi-Williams distribution (CWD), and the continuous wavelet transform (CWT), we prefer and propose using the CWT since it possesses frequency-dependent windowing, which allows for the arbitrary resolution of the signal frequency components to be obtained [19]. Also, the ratio of red CWT modulus to IR CW'T modulus was shown to 
relate to the $\% \mathrm{SpO}_{2}$ [20]. Details of the proposed processing methods and the experiments are provided in the rest of this paper in more detail.

\section{Materials and Methods}

\subsection{The Data Acquisition}

We designed and constructed the PPG acquisition system in our laboratory to detect the red and IR PPGs for the experiments. There were two measurement channels so that both the red and IR PPGs can be measured at two different positions simultaneously. For convenience, we used two commercial pulse oximeter probes. The wavelengths of light emitted from the light source inside each probe were measured using a spectrometer, USB4000-UV-VIS Ocean Optics. As shown in Figs. 1(a) and 1(b), the peak wavelengths of light emitted from the light source inside each probe are $659 \mathrm{~nm}$ and $914 \mathrm{~nm}$ for probe 1, and $659 \mathrm{~nm}$ and $920 \mathrm{~nm}$ for probe 2, respectively.

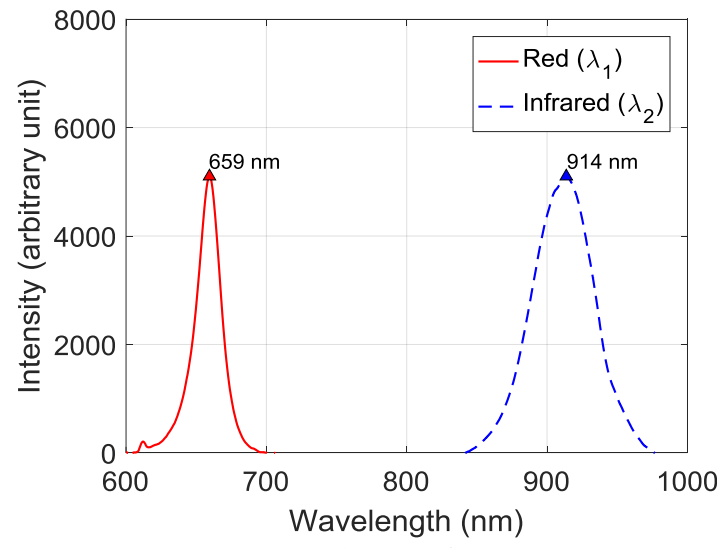

a) Probe 1

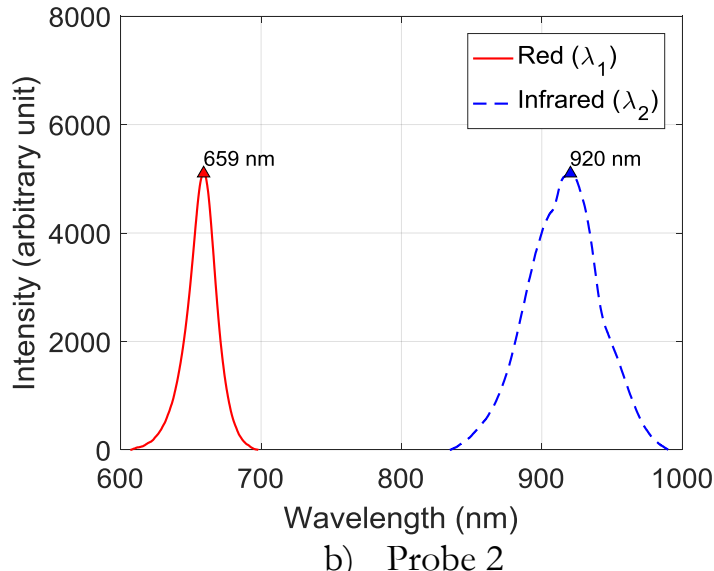

b) Probe 2

Fig. 1. The peak wavelengths emitted from the light source inside each probe.

All the detected PPGs were converted into a digital format using 12-bit analog-to-digital converters, and sent to a personal computer for recording and post-processing with MATLAB. The sampling frequency is $50 \mathrm{~Hz}$.

A calibration was performed in order to derive the appropriate calibration curve for measuring the $\% \mathrm{SpO}_{2}$ with each probe using the Fluke Biomedical $\% \mathrm{SpO}_{2}$ Simulator Index 2, working as an artificial finger. The calibration curve provides the relationship between the $\% \mathrm{SpO}_{2}$ and the ratio $r_{a}$, which is the ratio of red absorption to IR absorption due to the arterial blood. The $\% \mathrm{SpO}_{2}$ defined at the simulator and the averaged ratio $r_{a}$ derived from the measurement were fitted into a second-order polynomial equation. Equations (1) and (2) are the calibration equations appropriate for measuring $\% \mathrm{SpO}_{2}$ with probe 1 and 2, respectively. The plots of the $\% \mathrm{SpO}_{2}$ versus the averaged ratio $r_{a}$ and their fitted calibration curves are shown in Fig. 2.

$$
\begin{aligned}
& \text { Probe 1: } \% \mathrm{SpO}_{2}=-23.90 r_{a}{ }^{2}-6.17 r_{a}+109.29 \\
& \text { Probe 2: } \% \mathrm{SpO}_{2}=-22.58 r_{a}{ }^{2}-4.64 r_{a}+107.90
\end{aligned}
$$




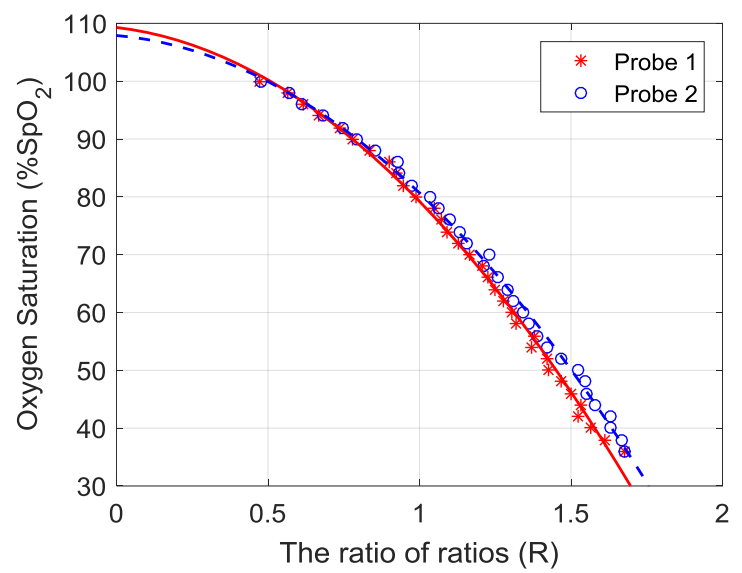

Fig. 2. The calibration curves appropriate for each probe.

\subsection{The PPG Model}

PPG measurement is normally accomplished by emitting light from the light source to the biological tissue, and detecting the light travelling through the tissue by either reflectance or transmittance mode. In the CPO, the detected PPG is modelled by considering that the light absorbers have two main groups; pulsatile and non-pulsatile tissues. According to the Beer-Lambert's law, the PPG can be modeled as follows:

$$
I^{\lambda}(t)=I_{0}^{\lambda} e^{-A_{a c}(t)} e^{-A_{d c}}+n(t)
$$

where $I^{\lambda}(t)$ is the detected light intensity, $I_{0}^{\lambda}$ is the light intensity emitted from the light source, $A_{a c}(t)$ and $A_{d c}$ represent the light absorption due to the pulsatile and non-pulsatile tissues, respectively, and $n(t)$ is the additive noise. Under the assumption that the pulsatile tissue is the arterial blood, which contains only the oxyhaemoglobin $\left(\mathrm{HbO}_{2}\right)$ and deoxyhaemoglobin $(\mathrm{Hb})$, the PPG model can therefore be written as follows:

$$
I^{\lambda}(t)=I_{0}^{\lambda} e^{-\left(\varepsilon_{H b O_{2}}^{\lambda}\left[H_{b O}\right]_{a}+\varepsilon_{H b}^{\lambda}[H b]_{a}\right) L_{a}^{\lambda}(t)} e^{-A_{d c}}+n(t)
$$

where $\varepsilon_{\mathrm{HbO}_{2}}^{\lambda}$ and $\varepsilon_{\mathrm{Hb}}^{\lambda}$ represent the extinction coefficient of $\mathrm{HbO}_{2}$ and $\mathrm{Hb}$ at the wavelength $\lambda$, $\left[\mathrm{HbO}_{2}\right]_{a}$ and $[H b]_{a}$ represent the concentration of $\mathrm{HbO}_{2}$ and $\mathrm{Hb}$ in the arterial blood, respectively, and $L_{a}^{\lambda}(t)$ represents the optical path length of light in the arterial blood at the wavelength $\lambda$. The $L_{a}^{\lambda}(t)$ varies with the arterial blood volume changing with the cardiac pulsation.

Equation (4) is the conventional PPG model that generally appears in the pulse oximetry context, and is widely used in $\mathrm{CPO}$ processing to determine the $\mathrm{SSpO}_{2}$. However, this model works well only when the PPG is not contaminated with the MA. The $A_{d c}$ is no longer constant when the body is moved. The $A_{d c}$ becomes $A_{d c}(t)$ and an error occurs because the CPO processing misinterprets $A_{d c}$ as $A_{a c}(t)$.

Presumably, the MA is caused by multiple factors, such as the movement of the sensor relative to the skin, deformation of tissues due to external pressure, and changes of haemodynamics. These factors directly affect the optical path length between the light source and detector, making the MA contaminated in the PPG. In this study, the conventional PPG model was revised by adding a term describing the attenuation of light caused by the motion as follows:

$$
I^{\lambda}(t)=I_{0}^{\lambda} e^{-\left(\varepsilon_{H b O_{2}}^{\lambda}\left[\mathrm{HbO}_{2}\right]_{a}+\varepsilon_{H b}^{\lambda}[\mathrm{Hb}]_{a}\right) L_{a}^{\lambda}(t)} e^{-A_{d c}} e^{-\mu_{\text {motion }}^{\lambda} L_{\text {motion }}^{\lambda}(t)}+n(t)
$$

where $\mu_{\text {motion }}^{\lambda}$ and $L_{\text {motion }}^{\lambda}(t)$ represent the absorption coefficient of the non-pulsatile tissues and the optical path length in the non-pulsatile tissues that varies during the body movement, respectively. Among the non-pulsatile tissues, the venous blood is considered to be the main component that changes the most during movement. We consider the term $\mu_{\text {motion }}^{\lambda} L_{\text {motion }}^{\lambda}(t)$ and its derivative as the MA. 


\section{3. $\% \mathrm{SpO}_{2}$ Estimation Method}

The block diagram of our proposed processing method is shown in Fig. 3. The operation and description of each block is described below.

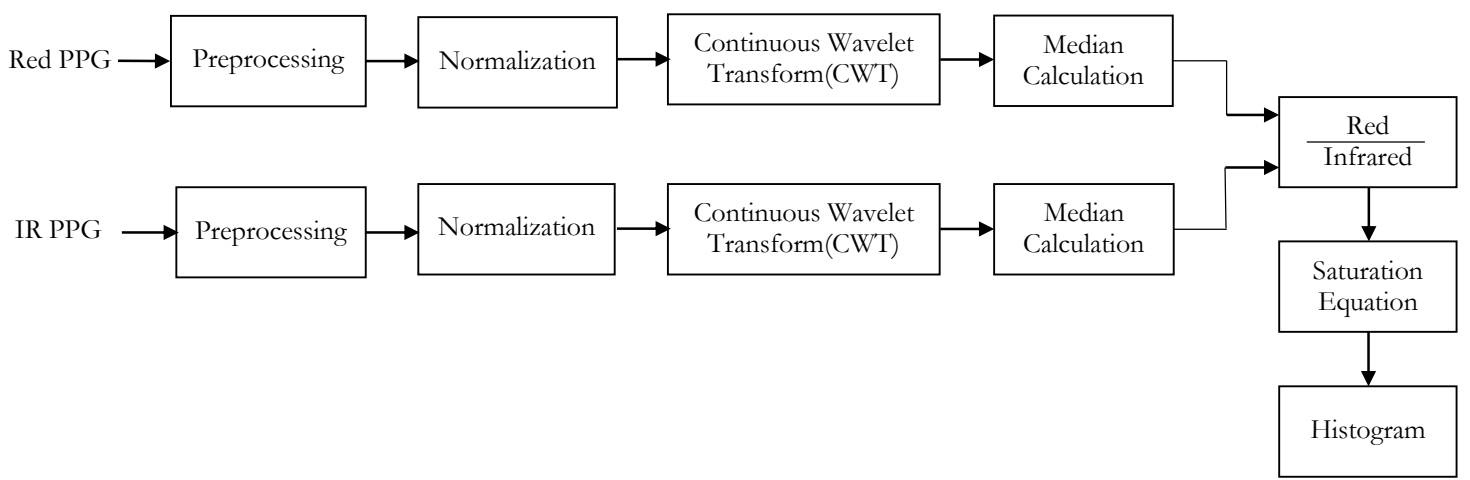

Fig. 3. Block diagram of the proposed processing method.

\subsubsection{Preprocessing}

A main objective of the preprocessing is to eliminate the noise and MA outside the frequency band of interest. On the understanding that the maximum human pulse is 240 beats per minute (BPM), or approximately 4 $\mathrm{Hz}$, the raw PPG was preprocessed using a low-pass filter with the cutoff frequency of approximately $5 \mathrm{~Hz}$, in order to cover the maximum desired frequency. We propose applying the Savitzky-Golay (SG) filter, a least-squares polynomial smoothing filter [21], for use as the low pass filter. The SG filter can provide the smoothed PG and its first derivative simultaneously, which are required for the normalization process. After preprocessing, the detected PPG can be described by the following equation:

$$
I^{\lambda}(t)=I_{0}^{\lambda} e^{-\left(\varepsilon_{\mathrm{HbO}}^{\lambda}\left[\mathrm{HbO}_{2}\right]_{a}+\varepsilon_{\mathrm{Hb}}^{\lambda}[\mathrm{Hb}]_{a}\right) L_{a}^{\lambda}(t)} e^{-A_{d c}} e^{-\mu_{\text {motion }}^{\lambda} L_{\text {motion }}^{\lambda}(t)}
$$

\subsubsection{Normalization}

Normalization is a process used to separate the light absorption of the pulsatile tissues from the non-pulsatile tissues. In principle, the normalization can be performed in several ways, such as dividing by the maximum intensity, dividing by the average intensity, the derivative method, and the logarithmic method. In this study, we prefer and propose using the derivative method because of its simplicity. No additional algorithms, such as peak detection and/or interpolation, are needed in order to perform the normalization. The normalization with the derivative method provides the normalized first derivative PPG as follows:

$$
-\frac{\left(d I^{\lambda}(t) / d t\right)}{I^{\lambda}(t)}=\left(\varepsilon_{\mathrm{HbO}_{2}}^{\lambda}\left[\mathrm{HbO}_{2}\right]_{a}+\varepsilon_{\mathrm{Hb}}^{\lambda}[H b]_{a}\right) \frac{d L_{a}^{\lambda}(t)}{d t}+\mu_{\text {motion }}^{\lambda} \frac{d L_{\text {motion }}^{\lambda}(t)}{d t}
$$

As seen in Eq. (7), the normalization process transforms the MA from the multiplicative form into the additive form. When there is no motion $\left(d L_{\text {motion }}^{\lambda}(t) / d t=0\right)$, the normalized first derivative PPG provides a term resulting from the arterial light absorption. When there is motion $\left(d L_{\text {motion }}^{\lambda}(t) / d t \neq 0\right)$, it provides terms, which are the combination of the arterial light absorption and the MA.

Generally, two wavelengths of light, i.e. red and infrared, are required in pulse oximetry processing. Under the assumption that the variation of the optical path length is equal for both wavelengths, the PPG model for pulse oximetry processing can then be expressed as follows:

$$
\begin{aligned}
& x_{1}(t)=a_{11} s(t)+a_{12} m(t) \\
& x_{2}(t)=a_{21} s(t)+a_{22} m(t)
\end{aligned}
$$


where $x_{1}(t)$ and $x_{2}(t)$ represent the normalized first derivative of the red and IR PPG. $s(t)$ and $m(t)$ represent the first derivative of changes in the optical path length in the pulsatile and non-pulsatile tissues, respectively. $a_{11}$ and $a_{21}$ represent the absorption coefficient of the arterial blood at the wavelength $\lambda_{1}$ and $\lambda_{2}$, respectively. $a_{12}$ and $a_{22}$ represent the absorption coefficient of the non-pulsatile tissues, i.e. the $\mu_{\text {motion }}^{\lambda_{1}}$ and $\mu_{\text {motion }}^{\lambda_{2}}$, respectively.

The main objective of pulse oximetry processing is to determine the $\% \mathrm{SpO}_{2}$ by finding the ratio of the arterial light absorption between two wavelengths $\left(r_{a}\right)$, which is the ratio of $a_{11}$ to $a_{21}$. Using Eq. (8) and (9), this ratio can be determined as follows:

$$
r_{a}=\frac{a_{11}}{a_{21}}=\frac{\left(\varepsilon_{H b O_{2}}^{\lambda_{1}}\left[\mathrm{HbO}_{2}\right]_{a}+\varepsilon_{\mathrm{Hb}}^{\lambda_{1}}[\mathrm{Hb}]_{a}\right)}{\left(\varepsilon_{\mathrm{HbO}}^{\lambda_{2}}\left[\mathrm{HbO}_{2}\right]_{a}+\varepsilon_{\mathrm{Hb}}^{\lambda_{2}}[\mathrm{Hb}]_{a}\right)}
$$

Since the definition of functional haemoglobin saturation $\left(\mathrm{SaO}_{2}\right)$ is

$$
\mathrm{SaO}_{2}=\frac{\mathrm{HbO}_{2}}{\mathrm{HbO}_{2}+\mathrm{Hb}}
$$

the ratio $r_{a}$ is determined as follows:

$$
r_{a}=\frac{a_{11}}{a_{21}}=\frac{\left(\varepsilon_{\mathrm{HbO}_{2}}^{\lambda_{1}} \mathrm{SaO}_{2}+\varepsilon_{\mathrm{Hb}}^{\lambda_{1}}\left(1-\mathrm{SaO}_{2}\right)\right)}{\left(\varepsilon_{\mathrm{HbO}_{2}}^{\lambda_{2}} \mathrm{SaO}_{2}+\varepsilon_{\mathrm{Hb}}^{\lambda_{2}}\left(1-\mathrm{SaO}_{2}\right)\right)}
$$

Note that $\mathrm{SaO}_{2}$ is changed to $\mathrm{SpO}_{2}$ to emphasize that it is obtained from the pulse oximetry. After rearranging the terms, we obtain the theoretical relationship between the $\% \mathrm{SpO}_{2}$ and the ratio $r_{a}$ as

$$
\% \mathrm{SpO}_{2}=\frac{\left(\varepsilon_{H b}^{\lambda_{1}}-\varepsilon_{H b}^{\lambda_{2}}\right) r_{a}}{\varepsilon_{H b}^{\lambda_{1}-\varepsilon_{H b O_{2}}^{\lambda_{2}}+\left(\varepsilon_{H b O_{2}}^{\lambda_{2}}-\varepsilon_{H b}^{\lambda_{2}}\right) r_{a}}} \times 100
$$

In practice, this relationship is obtained from the calibration. The calibration equation used in this study is described in the previous section.

The ratio of light absorption of the non-pulsatile tissues $\left(r_{v}\right)$, which is the ratio of $a_{12}$ to $a_{22}$, only appears when the MA is present. As the venous blood is considered to be the main component that varies during movement, and the venous oxygen saturation is normally lower than the arterial oxygen saturation, the ratio $r_{v}$ is theoretically higher than $r_{a}$.

\subsubsection{Continuous wavelet transform and median calculation}

According to the pulse oximetry model in Eq. (8) and (9), when the ratios $r_{a}$ and $r_{v}$ are assumed to be constant over the time interval of interest, the frequency domain representation of the pulse oximetry model in Eq. (8) and (9) can thus be expressed as

$$
\begin{aligned}
& X_{1}(f)=a_{11} S(f)+a_{12} M(f) \\
& X_{2}(f)=a_{21} S(f)+a_{22} M(f)
\end{aligned}
$$

where $X_{1}(f), X_{2}(f), S(f)$, and $M(f)$ are the frequency domain representations of $x_{1}(t), x_{2}(t), s(t)$, and $m(t)$ respectively.

The main objective of this stage is to derive the frequency spectrum of the normalized first derivative PPG corresponding to Eq. (14) and (15). Instead of using the FFT, we propose applying TF analysis, which can be accomplished by using the CWT together with the median calculation.

After the normalization process, we segmented the normalized first derivative red and IR PPG into time windows. Each window had a six second interval and was updated by shifting the window along the time 
axis every one second, resulting in an overlap of five seconds. The CWT of the normalized first derivative PPG is defined as [19]

$$
\left(T_{i}(\alpha, \beta)=\frac{1}{\sqrt{\alpha}} \int_{-\alpha}^{\alpha} x_{i}(t) \psi^{*}\left(\frac{t-\beta}{\alpha}\right) d t\right.
$$

where $x_{i}(t)$ is the normalized first derivative PPG in each time window, $\psi^{*}(t)$ is the complex conjugate of the mother wavelet function $\psi(t), \alpha$ is the dilation parameter of the wavelet and $\beta$ is its location parameter. The Morlet function, one of the most popular wavelets used for the biosignal analysis, was chosen to be the mother wavelet, as it was formerly shown to be effective for pulse oximetry processing [20]. The Morlet wavelet is defined as

$$
\psi(t)=\frac{1}{\sqrt[4]{\pi}} e^{i \omega_{0} t} e^{-\frac{t^{2}}{2}}
$$

where $\omega_{0}$ is the central frequency of the mother wavelet.

Then, the CWT modulus, $\left|T_{i}(\alpha, \beta)\right|$, at the specific $\alpha$ scale and $\beta$ location was computed and plotted into the TF plane. In principle, the wavelet $\alpha$ scale is inversely proportional to the frequency $(f)$ as follows:

$$
f=\frac{f_{c}}{\alpha}
$$

where $f_{c}$ is the characteristic frequency of the mother wavelet. From the TF plot, the median calculation along the time axis is proposed to determine the frequency spectrum of the normalized first derivative PPG.

\subsubsection{Finding the ratio of red to IR}

From the frequency spectrum of the normalized first derivative PPG obtained in the previous stage, the ratio of each red CWT modulus to each corresponding infrared CW'T modulus $(R(f))$ was computed at each frequency. Under the assumption that the frequency components of the cardiac pulsation $\left(f_{a}\right)$ are different from those of the motion artifact $\left(f_{v}\right)$, the ratio $R(f)$ can be written as the combination of $r_{a}$ and $r_{v}$ as follows:

$$
R(f)=\frac{a_{11} S\left(f_{a}\right)}{a_{21} S\left(f_{a}\right)}+\frac{a_{12} M\left(f_{v}\right)}{a_{22} M\left(f_{v}\right)}=r_{a}\left(f_{a}\right)+r_{v}\left(f_{v}\right)
$$

\subsubsection{Computing the the oxygen saturation and identifying the $\% \mathrm{SpO}_{2}$}

The ratio $\mathrm{R}(\mathrm{f})$ in each frequency was then converted to the oxygen saturation by using the saturation equation, i.e. Eq. (1) or (2) depending on the probe used. Only the frequency range from 0.5 to $5 \mathrm{~Hz}$ corresponding to a pulse rate of 30 to $300 \mathrm{BPM}$ is considered. The calibration curve in Figure 2 implies that the higher the ratio $R(f)$, the lower the oxygen saturation becomes. As mentioned earlier, the ratio $r_{v}$ appears only when there is the MA and it is normally higher than the ratio $r_{a}$. Therefore, there may be variation in the oxygen saturation, especially when the PPG is corrupted with the MA. In order to identify the $\% \mathrm{SpO}_{2}$ among the saturation data, all of the saturation data was plotted on a histogram. From the graphical representation of the histogram, it was interpreted that a bin having the highest level of oxygen saturation and the maximum number of saturation data represents the $\% \mathrm{SpO}_{2}$. 


\subsection{Experiments}

Experiments were held to evaluate the proposed method in processing both the clean PPG and the motioncorrupted PPG for determining the $\% \mathrm{SpO}_{2}$. The developed PPG acquisition system was used to detect the red and IR PPGs from a healthy human subject at two different positions, simultaneously. A probe connected to channel 1 was clipped on the left index finger, while another was connected to channel 2 and clipped on the right index finger. The MA was generated by bending the finger. The measurement consists of 3 protocols. For the first protocol, both fingers were stationary. The second protocol was divided into 3 states; resting, moving, and resting, respectively. In the resting states, both fingers were stationary. In the moving stage, the left index finger remained stationary, while the right index finger performed a transient finger bend. The third protocol is similar to the second protocol, except that the right index finger was periodically bent in the moving stage.

All the detected PPG signals were then processed using both the proposed method and the CPO. The $\% \mathrm{SpO}_{2}$ obtained from the proposed method was compared to that from the $\mathrm{CPO}$, and also the $\% \mathrm{SpO}_{2}$ measured from the left index finger was compared to that from the right index finger.

For the CPO processing, the ratio $r_{a}$ was computed based on using the linear regression analysis (LRA) as follows [22]:

$$
r_{a}=\frac{n \sum x_{i} y_{i}-\sum x_{i} \sum y_{i}}{n \sum x_{i}^{2}-\left(\sum x_{i}\right)^{2}}
$$

where $x$ and $y$ represent the normalized first derivative red and IR PPG, respectively, $i$ represents the sample number, and $n$ represents the number of samples. Then, the ratio $r_{a}$ was converted to the $\% \mathrm{SpO}_{2}$ by using Eq. (1) or (2) depending on the probe used.

\subsection{Performance Measurement}

The Bland-Altman analysis [23], which is a statistical method for comparing two different measurement techniques, was used to compare the $\% \mathrm{SpO}_{2}$ measured from both methods and positions. The bias is defined as the mean difference between the two methods $(\bar{d})$, the precision is defined as the standard deviation $(s)$ of differences, and the limits of agreement (LOA) indicating the boundary of the $95 \%$ of difference is defined as $\bar{d} \pm 1.96 \mathrm{~s}$. As the left index finger was stationary in all measurement protocols, the $\% \mathrm{SpO}_{2}$ obtained from the CPO at channel 1 was used as a reference. An LOA of less than $\pm 3 \%$ is considered to be clinically acceptable [24].

\section{Results and Discussion}

Figure 4 provides an example of applying the proposed processing method to the clean PPG, while Figs. 5 and 6 depict examples of applying the proposed processing method to the motion corrupted PPG, of which the MA was induced by a transient finger bend and the periodic finger bend, respectively. Each figure is composed of 7 subfigures identified as " $a$ " to " $\mathrm{g}$ ".

Figure (a) shows the red and IR PPG after being preprocessed with the SG filter. The normalization with the derivative method provided the normalized first derivative red and IR PPG, of which the waveform indicates the arterial blood flow rather than the arterial blood volume, as shown in Fig. (b). Its physical meaning in Eq. (7) reveals that it can be used to represent the arterial light absorption and can be further processed for determining the $\% \mathrm{SpO}_{2}$. The CWT decomposed the normalized first derivative PPG, and mapped into the TF plane. The TF plots of the CWT modulus of the normalized first derivative red and IR PPG are shown in Fig. (c) and (d), respectively. The median calculation along the time axis provided the frequency spectra of the normalized first derivative red and IR PPG, as shown in Fig. (e). As the resolution of the wavelet $\alpha$ scale can be arbitrarily defined, the spectral resolution of the frequency spectrum obtained from this method is greater than using the FFT. The median calculation also helps to eliminate the impact of the transient MA. Figure 5(e) shows that the peak of the frequency spectrum corresponding to the transient finger bend was reduced as a result of the median calculation. However, the median calculation cannot eliminate the frequency components induced by the periodic movement, as shown in Fig. 6(e). 
Figure ( $\mathrm{f}$ ) shows the oxygen saturation at each frequency. When there is no MA, the oxygen saturation at each frequency is quite consistent as shown in Fig. 4(f). Because of the effects of the MA, the oxygen saturation at some frequencies deviates in the lower direction, as shown in Fig. 5(f) and 6(f). The histogram of the oxygen saturation data is shown in Fig. $(\mathrm{g})$. When there is no MA, all saturation data correspond to the ratio $r_{a}$ and represents the $\% \mathrm{SpO}_{2}$, as shown in Fig. 4(g). The MA causes changes in the distribution of the saturation data. The saturation data corresponding to the ratio $r_{v}$ are distributed separately from those corresponding to the ratio $r_{a}$. However, the $\% \mathrm{SpO}_{2}$ corresponding to the ratio $r_{a}$ can still be identified, as shown in Figs. 5(g) and 6(g).

The examples of results of using the CPO and the proposed method to process the clean PPG, and the PPG corrupted with the transient and periodic MA, are shown in Figure 7, 8, and 9, respectively. The result obtained from the left index finger was considered as reference, and it was compared to that from the right index finger.

Figure 7 reveals that, when there was no MA, the $\% \mathrm{SpO}_{2}$ obtained from both methods and positions was quite similar. For the $\mathrm{CPO}$, the slopes of linear regression lines, which relate to the ratio $r_{a}$ and the $\% \mathrm{SpO}_{2}$, are quite coincident, as shown in Fig. 7(a). The ratio $r_{a}$ measured from the left and right index finger are 0.6116 and 0.6188 , corresponding to the $\% \mathrm{SpO}_{2}$ at $96.9 \%$ and $96.5 \%$, respectively. For the proposed method, the peaks of histograms that indicate the $\% \mathrm{SpO}_{2}$ are almost overlap. As shown in Fig. $7(\mathrm{~b})$, the $\% \mathrm{SpO}_{2}$ measured from the left and right index finger are $96.6 \%$ and $96.4 \%$, respectively.

Figure 8 and 9 show that the $\mathrm{CPO}$ is sensitive to the MA, but the proposed method has resistance to the MA. The CPO tends to provide the false desaturation, when the MA is present. The slope of the linear regression line tends to be increased, when the detected PPGs were corrupted with the transient or periodic $\mathrm{MA}$. Because of the $\mathrm{MA}$, the $\% \mathrm{SpO}_{2}$ measured from the right index finger was, therefore, lower than that from the left index finger as shown in Fig. 8(a) and 9(a). The proposed method has more resistant to the transient and periodic MA than the CPO. As shown in Fig. 8(b) and 9(b), although the MA makes changes in the distribution of the saturation data in the histogram, the $\% \mathrm{SpO}_{2}$ can still be specified by a peak of the highest level of oxygen saturation.

Figure 10 depicts the results of the $\% \mathrm{SpO}_{2}$ measurements at channels 1 and 2. Figure 10(a) shows the normalized first derivative red and IR PPGs detected from the left index finger at channel 1 without the MA. Figure 10(b) is the normalized first derivative red and IR PPG detected from the right index finger at channel 2 with the MA induced by the periodic finger bend. Figures 10 (c) and $10(\mathrm{~d})$ show the $\% \mathrm{SpO}_{2}$ obtained from the proposed method and the $\mathrm{CPO}$ at channels 1 and 2, respectively. Figure 10 (c) reveals that when there is no MA, the proposed processing method can provide the $\% \mathrm{SpO}_{2}$ similar to the CPO. As shown in Fig. 10 (d), the CPO provides a false desaturation when the red and IR PPGs are contaminated with the MA, while the $\% \mathrm{SpO}_{2}$ obtained from the proposed method remains consistent. These results reveal that the proposed method is more resistant to the MA than the CPO.

Figure 11 is the Bland-Altman plot comparing the $\% \mathrm{SpO}_{2}$ obtained from the proposed processing method at channel 2 versus that from the CPO measured at channel 1 when there is no MA. There were 500 samples $(n)$ of analyzed data. The bias is 0.01 , and the LOA is in the range of -0.90 to 0.92 . It can be interpreted that the proposed method can provide the $\% \mathrm{SpO}_{2}$ similar to the $\mathrm{CPO}$ when there is no MA. On the other hand, the $\% \mathrm{SpO}_{2}$ obtained from the proposed method can be used interchangeably with that from the $\mathrm{CPO}$ when there is no $\mathrm{MA}$, and the $\% \mathrm{SpO}_{2}$ measured from the right index finger represents the arterial oxygen saturation similar to the $\% \mathrm{SpO}_{2}$ measured from the left index finger.

Tables 1 and 2 show the results of the Bland-Altman analysis comparing the $\% \mathrm{SpO}_{2}$ obtained from the $\mathrm{CPO}$ and the proposed method at channel 2 versus that from the $\mathrm{CPO}$ measured at channel 1 , when the MA was induced by the transient and periodic finger bend, respectively. The analyzed data for both measurement protocols included 110 samples. The results in both tables showed that the bias of the proposed method is less than that of the $\mathrm{CPO}$, indicating that the proposed method was more resistant to the MA than the CPO. However, the range of LOA was still quite wide. The impact of periodic MA to the proposed processing method would depend on both strength and frequency of the MA. The greater the strength of MA is greater, the larger the bias and the range of LOA becomes. If the MA can be partially eliminated from the PPG before applying the proposed processing method, the range of LOA would potentially be narrower and within the acceptable range. 


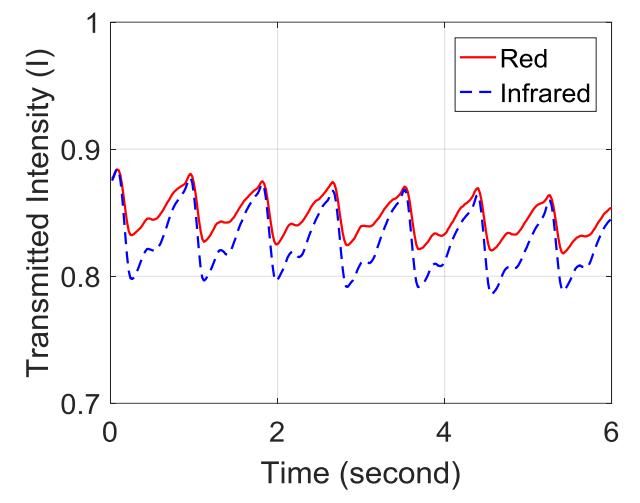

a) The preprocessed PPG

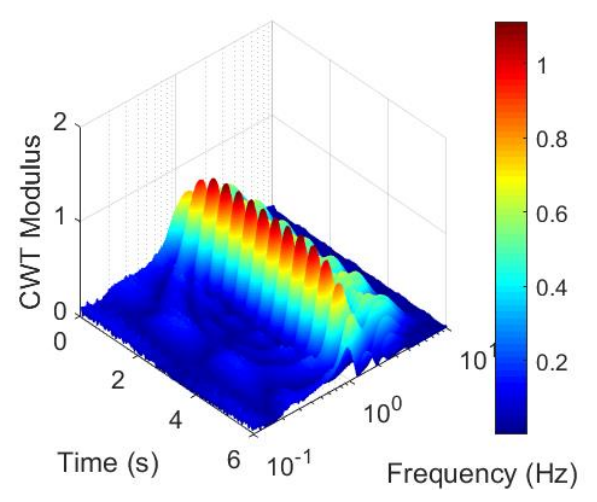

c) The TF plot of red signal.

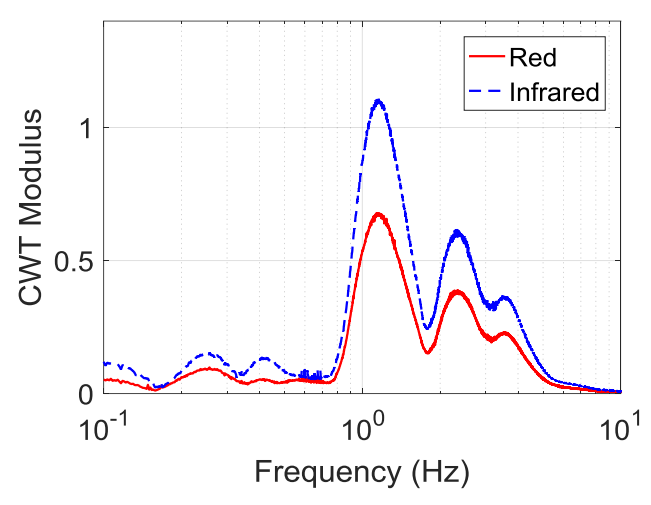

e) The frequency spectrum

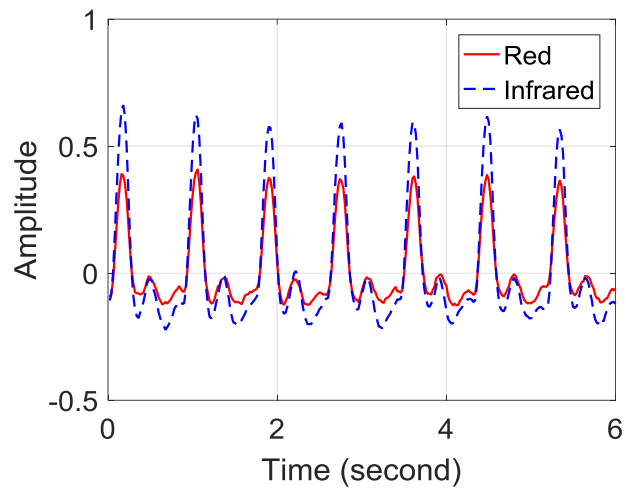

b) The normalized first derivative PPG

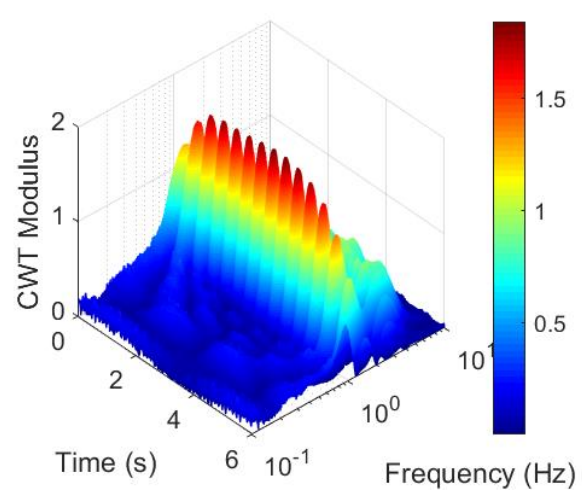

d) The TF plot of IR signal

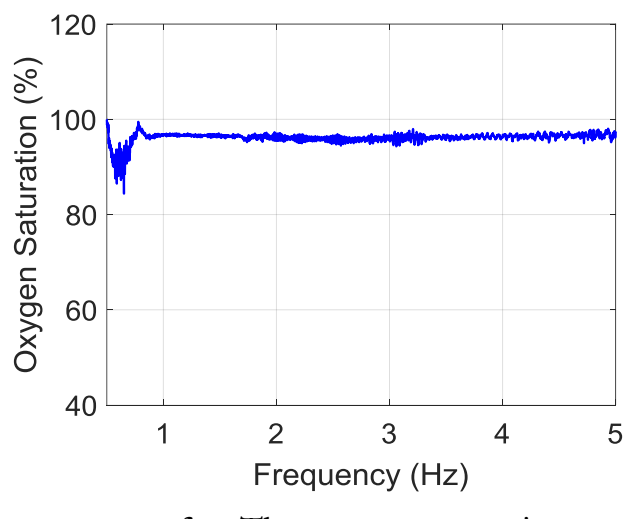

f) The oxygen saturation

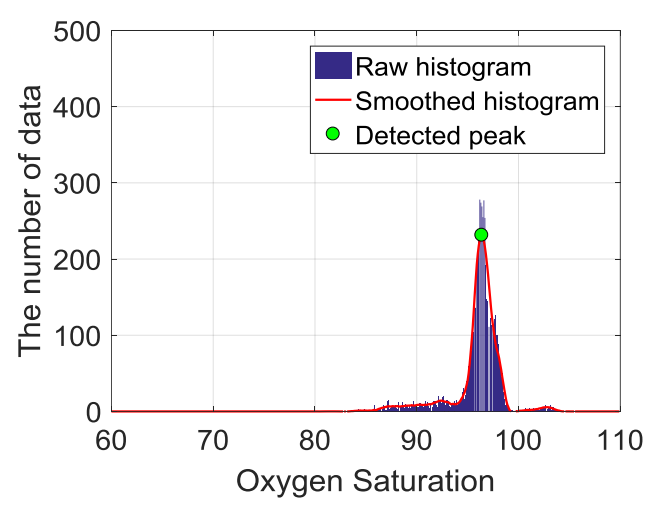

g) The histogram of the oxygen saturation

Fig. 4. Example of processing the clean PPG. 


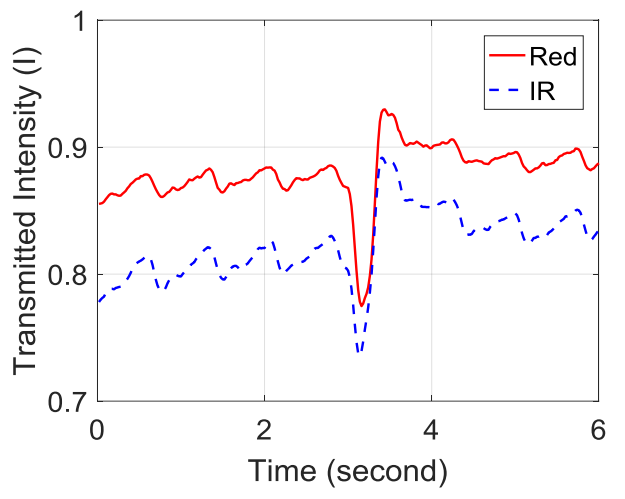

a) The preprocessed PPG

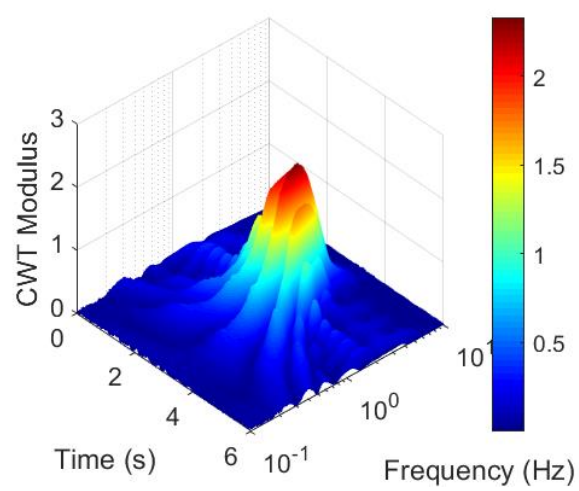

c) The TF plot of red signal.

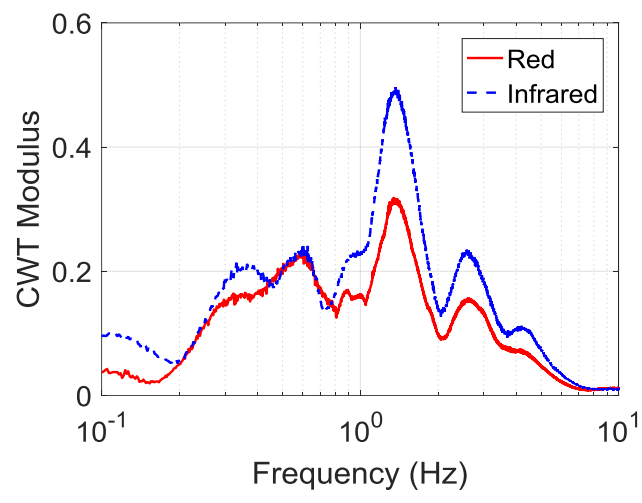

e) The frequency spectrum

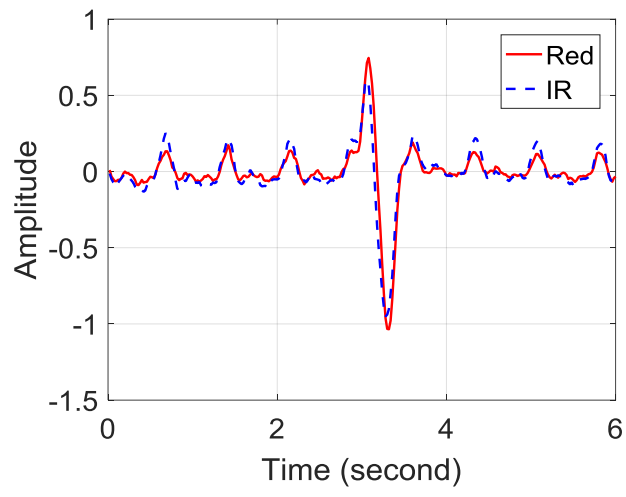

b) The normalized first derivative PPG

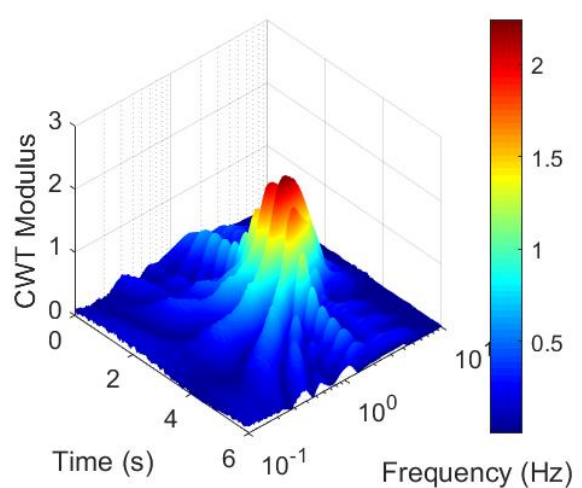

d) The TF plot of IR signal

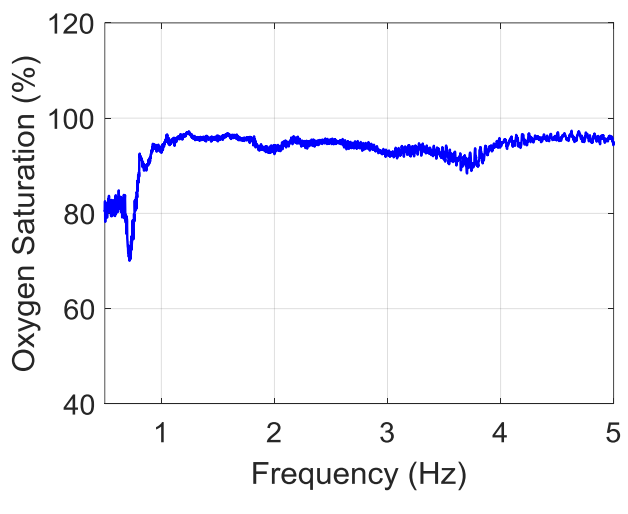

f) The oxygen saturation

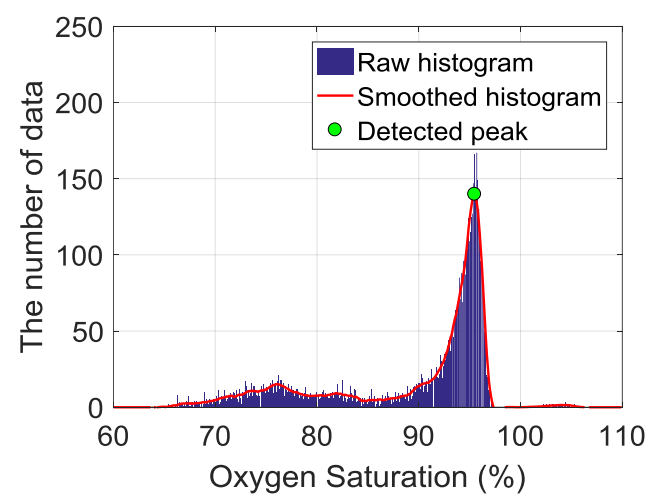

g) The histogram of the oxygen saturation

Fig. 5. Example of processing the PPG corrupted by a transient finger bend. 


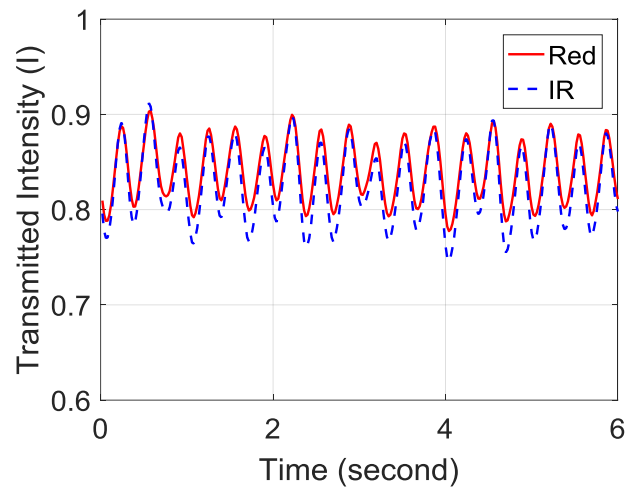

a) The preprocessed PPG

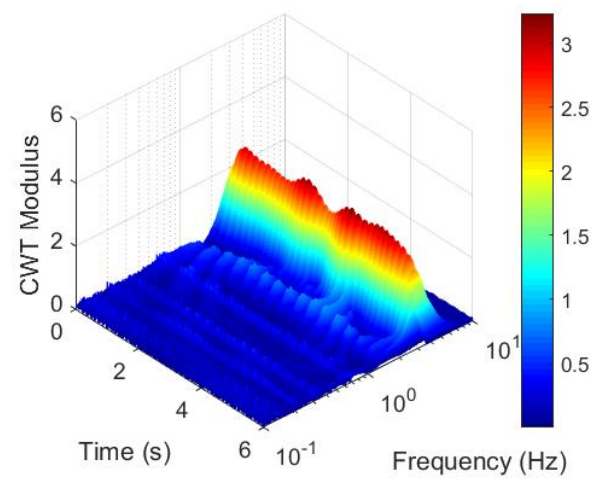

c) The TF plot of red signal.

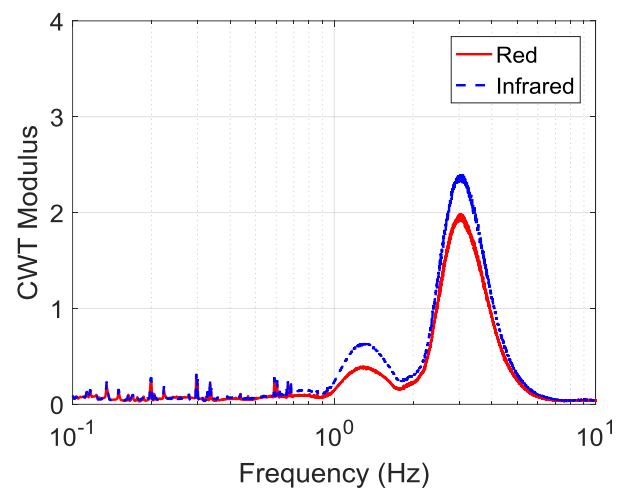

e) The frequency spectrum

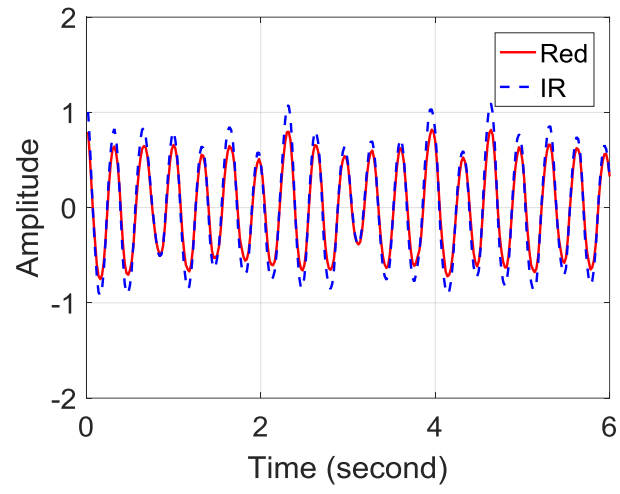

b) The normalized first derivative PPG

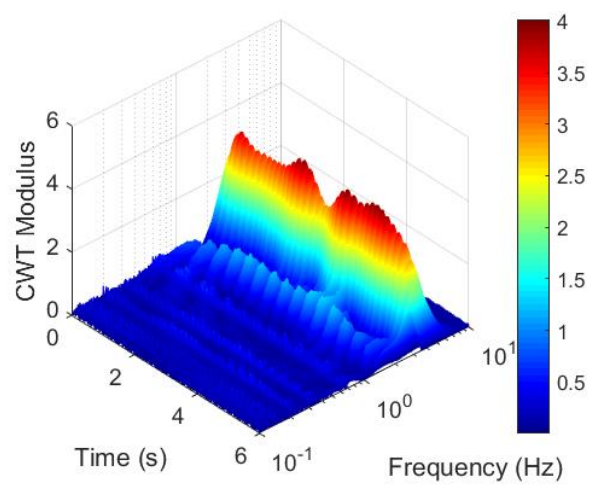

d) The TF plot of IR signal

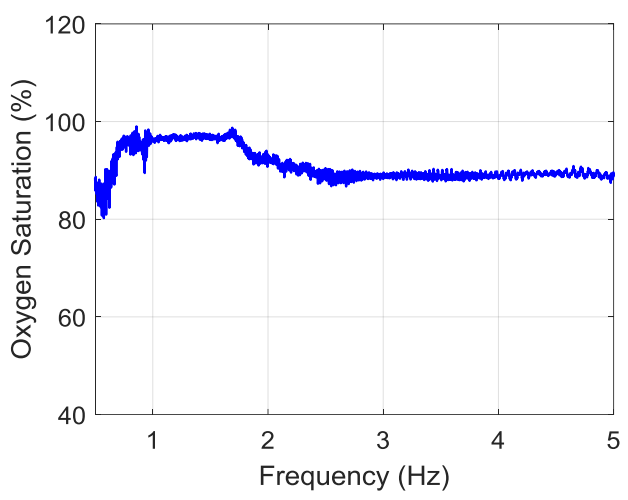

f) The oxygen saturation

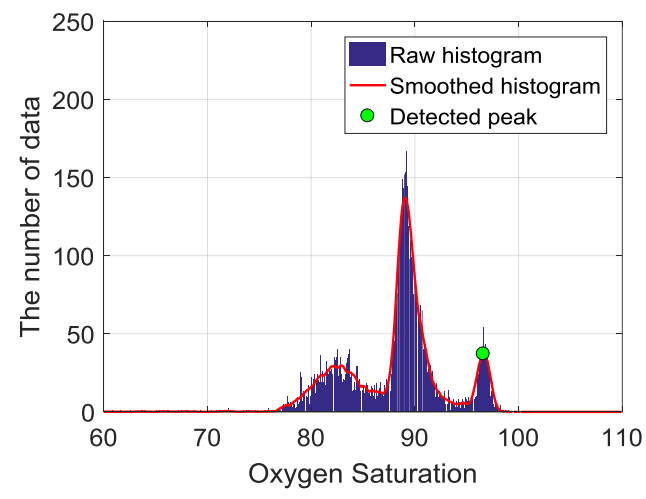

g) The histogram of the oxygen saturation

Fig. 6. Example of processing the PPG corrupted by the periodic finger bend. 


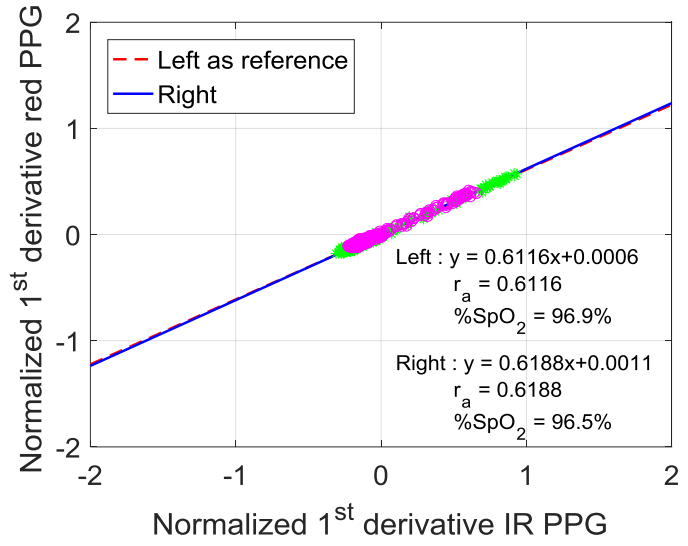

a) $\mathrm{CPO}$

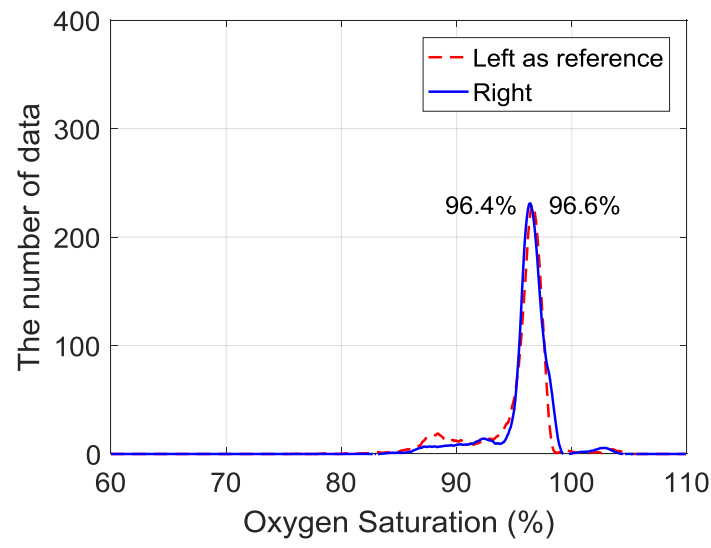

b) Proposed method

Fig. 7. Examples of results of using the CPO and the proposed method to process the clean PPG.

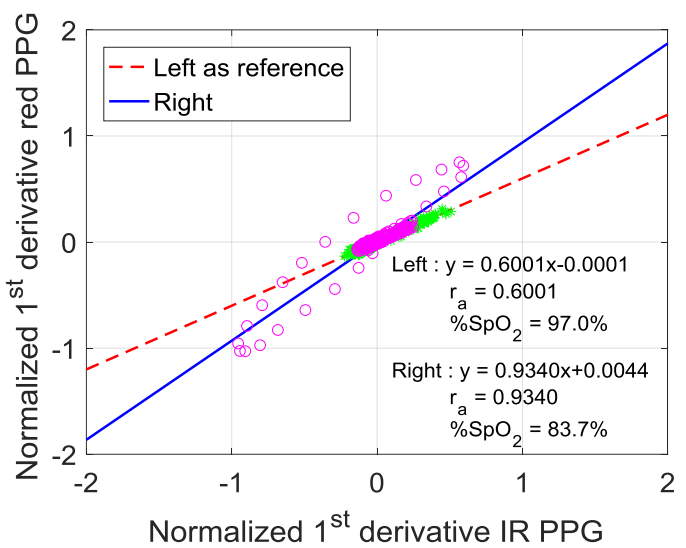

a) $\mathrm{CPO}$

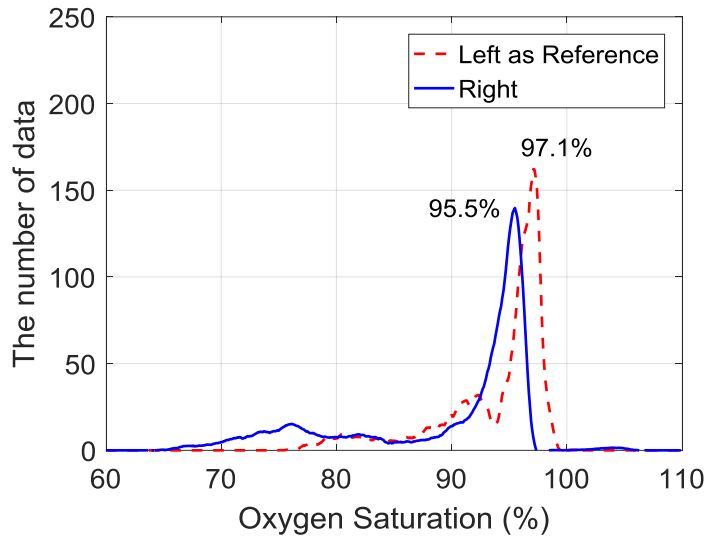

b) Proposed method

Fig. 8. Examples of results of using the CPO and the proposed method to process the PPG corrupted with the transient MA.

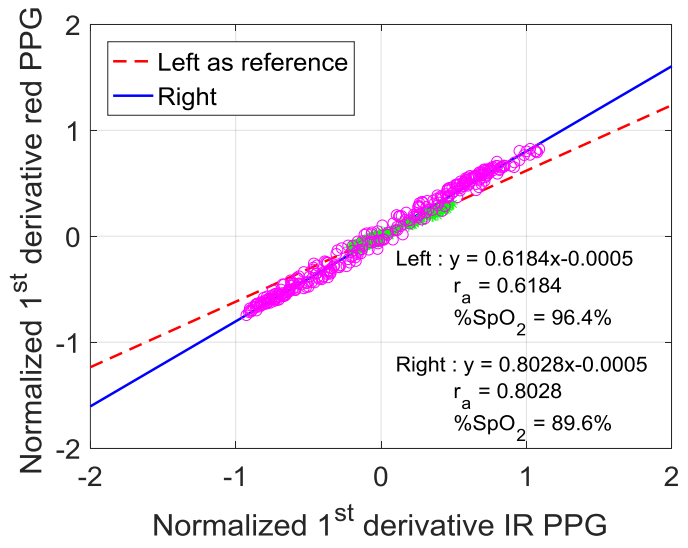

a) $\mathrm{CPO}$

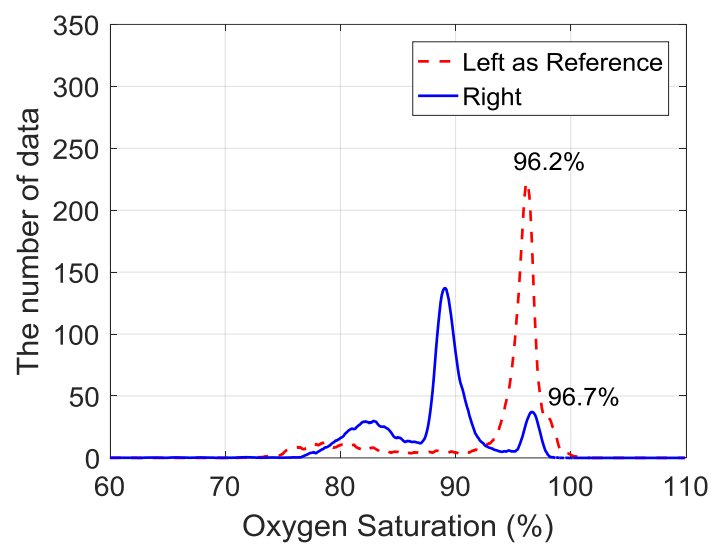

b) Proposed method

Fig. 9. Examples of results of using the CPO and the proposed method to process the PPG corrupted with the periodic MA. 


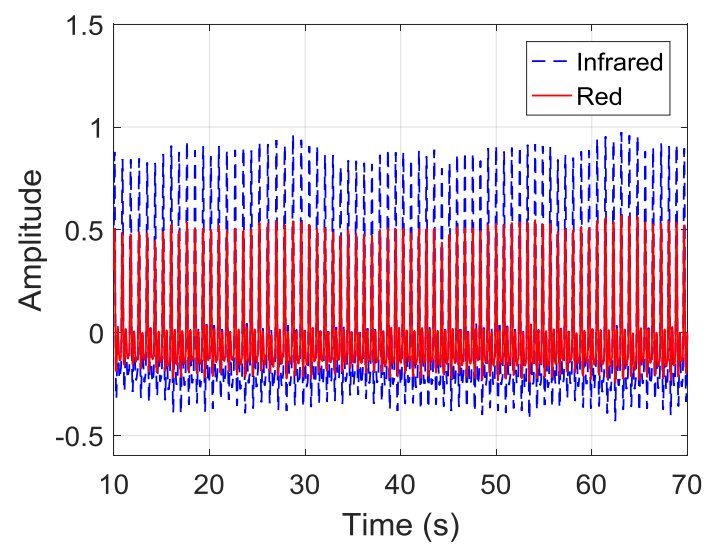

a) The normalize first derivative red and IR PPG measured from left index finger at channel 1

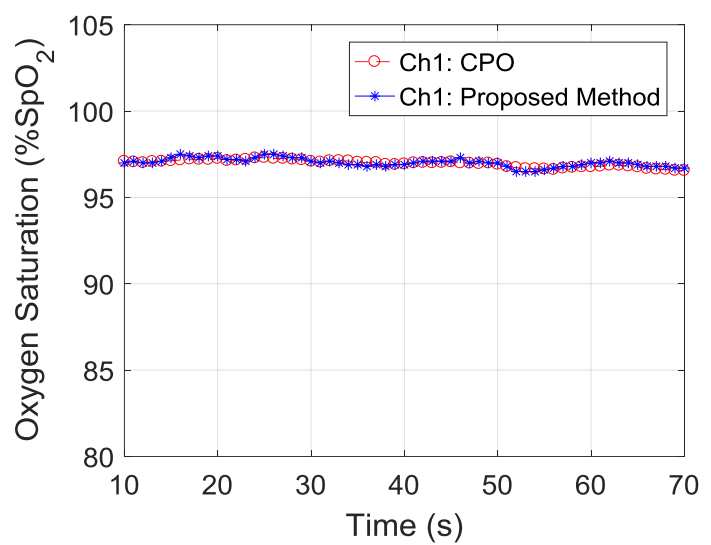

c) The $\% \mathrm{SpO}_{2}$ measured from left index finger at channel 1.

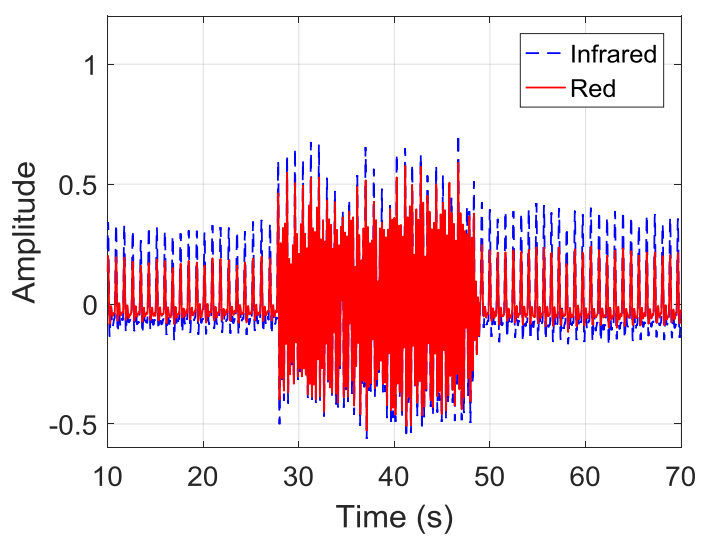

b) The normalize first derivative red and IR PPG measured from right index finger at channel 2

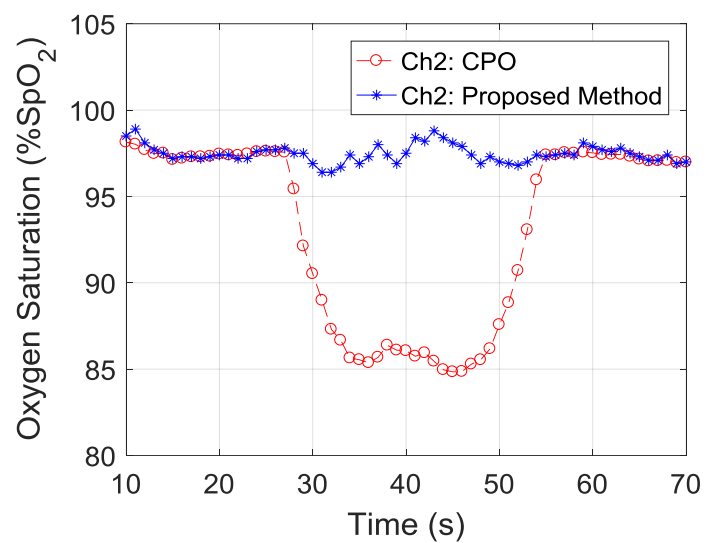

d) The $\% \mathrm{SpO}_{2}$ measured from right index finger at channel 2 .

Fig. 10. Example of the $\% \mathrm{SpO}_{2}$ measurement obtained from two different positions and two methods simultaneously

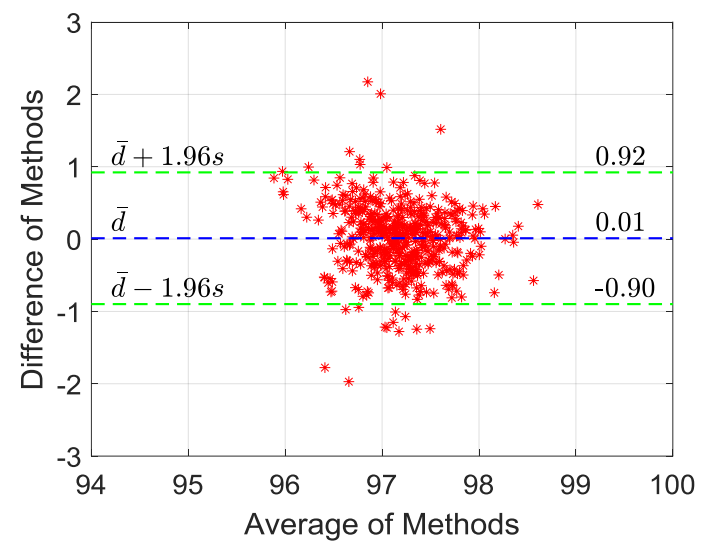

Fig. 11. The Bland-Altman plot showing the comparison between the $\% S p O_{2}$ obtained from the proposed method versus that from the CPO measured at channel 1 when there is no MA $(n=500)$. 
Table 1. The Bland-Altman analysis plot showing the comparison-between the $\% \mathrm{SpO}_{2}$ obtained from the $\mathrm{CPO}$ and the proposed method at channel 2 versus that from the $\mathrm{CPO}$ at channel 1 during transient finger bend $(n=110)$.

\begin{tabular}{lcc}
\hline & Ch 2: CPO & Ch 2: Proposed Method \\
& vs & vs \\
& -8.02 & Ch 1: CPO \\
\hline Bias $(\bar{d})$ & 3.09 & -1.01 \\
Precision $(s)$ & -1.97 & 1.20 \\
+ LOA $(\bar{d}+1.96 s)$ & -14.07 & 1.34 \\
- LOA $(\bar{d}-1.96 s)$ & -3.37 \\
\hline
\end{tabular}

Table 2. The Bland-Altman analysis between the $\% \mathrm{SpO}_{2}$ obtained from the $\mathrm{CPO}$ and the proposed method at channel 2 versus that from the CPO measured at channel 1 during periodic finger bend $(n=110)$.

\begin{tabular}{lcc}
\hline & Ch 2: CPO & Ch 2: Proposed Method \\
& vs & vs \\
& -9.53 & Ch 1 : CPO \\
\hline Bias $(\bar{d})$ & 1.20 & -0.82 \\
Precision $(s)$ & -4.17 & 1.96 \\
+ LOA $(\bar{d}+1.96 s)$ & -11.89 & 3.02 \\
- LOA $(\bar{d}-1.96 s)$ & -4.66 \\
\hline
\end{tabular}

\section{Conclusions}

In this paper, we proposed a motion resistant pulse oximetry processing based on time-frequency analysis. It was suggested that the SG filter be used in the preprocessing stage, because it can simultaneously provide both the smoothed PPG and its derivative, which are required for normalization. The normalization with the derivative method was recommended because of its simplicity in computation. However, it provided the normalized first derivative PPG indicating the arterial blood flow rather than the normalized PPG indicating the arterial blood volume. The CWT together with the median calculation was proposed for finding the frequency spectrum of the normalized first derivative red and IR PPG, instead of using the FFT. Using this method, the spectral resolution of the frequency spectrum can be arbitrarily increased, leading to an increase in the number of data points to be plotted on the histogram. Experimental results showed that the proposed processing method was more resistant to the MA than the CPO. The increased spectral resolution helped reveal the correct $\% \mathrm{SpO}_{2}$ more clearly, and made the estimation of the $\% \mathrm{SpO}_{2}$ more accurate, especially when there was a MA. As many signal processing techniques have been proposed to eliminate the MA, the pulse oximetry processing would be more powerful if those techniques are combined with the method proposed in this study.

\section{References}

[1] T. Aoyagi, "Pulse oximetry: its invention, theory, and future," Journal of Anesthesia, vol. 17, no. 4, pp. 259-266, 2003.

[2] J. W. Severinghaus, "Takuo Aoyagi: discovery of pulse oximetry," Anesth Analg, vol. 105, no. 6 Suppl, pp. S1-4, Dec. 2007.

[3] J. Allen, "Photoplethysmography and its application in clinical physiological measurement," Physiol Meas, vol. 28, no. 3, pp. R1-39, Mar. 2007.

[4] W. Tin and M. Lal, "Principles of pulse oximetry and its clinical application in neonatal medicine," Semin Fetal Neonatal Med, vol. 20, no. 3, pp. 192-7, Jun. 2015.

[5] S. DeMeulenaere, "Pulse oximetry: Uses and limitations," The Journal for Nurse Practitioners, vol. 3, no. 5, pp. 312-317, 2007.

[6] M. T. Petterson, V. L. Begnoche, and J. M. Graybeal, "The effect of motion on pulse oximetry and its clinical significance," Anesth Analg, vol. 105, no. 6 Suppl, pp. S78-84, Dec. 2007. 
[7] K. A. Reddy, B. George, and V. J. Kumar, "Use of fourier series analysis for motion artifact reduction and data compression of photoplethysmographic signals," IEEE Transactions on Instrumentation and Measurement, vol. 58, no. 5, pp. 1706-1711, 2009.

[8] H. H. Asada, J. Hong-Hui, and P. Gibbs, "Active noise cancellation using MEMS accelerometers for motion-tolerant wearable bio-sensors," in The 26th Annual International Conference of the IEEE Engineering in Medicine and Biology Society, 2004, vol. 1, pp. 2157-2160.

[9] P. Gibbs and H. H. Asada, "Reducing motion artifact in wearable bio-sensors using MEMS accelerometers for active noise cancellation," in Proceedings of the 2005, American Control Conference, 2005, vol. 3, pp. 1581-1586.

[10] L. B. Wood and H. H. Asada, "Noise cancellation model validation for reduced motion artifact wearable PPG sensors using MEMS accelerometers," in 2006 International Conference of the IEEE Engineering in Medicine and Biology Society, 2006, pp. 3525-3528.

[11] M. R. Ram, K. V. Madhav, E. H. Krishna, N. R. Komalla, and K. A. Reddy, "A novel approach for motion artifact reduction in PPG signals based on AS-LMS adaptive filter," IEEE Transactions on Instrumentation and Measurement, vol. 61, no. 5, pp. 1445-1457, 2012.

[12] F. Peng, Z. Zhang, X. Gou, H. Liu, and W. Wang, "Motion artifact removal from photoplethysmographic signals by combining temporally constrained independent component analysis and adaptive filter," Biomed Eng Online, vol. 13, p. 50, Apr. 24, 2014.

[13] B. S. Kim and S. K. Yoo, "Motion artifact reduction in photoplethysmography using independent component analysis," IEEE Transactions on Biomedical Engineering, vol. 53, no. 3, pp. 566-568, 2006.

[14] R. Krishnan, B. Natarajan, and S. Warren, "Two-stage approach for detection and reduction of motion artifacts in photoplethysmographic data," IEEE Transactions on Biomedical Engineering, vol. 57, no. 8, pp. 1867-1876, 2010.

[15] M. R. Ram, K. V. Madhav, E. H. Krishna, N. R. Komalla, K. Sivani, and K. A. Reddy, "ICA-based improved DTCWT technique for MA reduction in PPG signals with restored respiratory information," IEEE Transactions on Instrumentation and Measurement, vol. 62, no. 10, pp. 2639-2651, 2013.

[16] C. M. Lee and Y. T. Zhang, "Reduction of motion artifacts from photoplethysmographic recordings using a wavelet denoising approach," in IEEE EMBS Asian-Pacific Conference on Biomedical Engineering, 2003, pp. 194-195.

[17] J. M. Goldman, M. T. Petterson, R. J. Kopotic, and S. J. Barker, "Masimo signal extraction pulse oximetry," Journal of Clinical Monitoring and Computing, vol. 16, no. 7, pp. 475-483, 2000.

[18] K. D. Mohamed, K.-A. Esmaiel, M. E. Ibrahim, J. M. Rex, M. W. Walter, and A. S. Robert, Signal Processing Apparatus. United States, 2009.

[19] P. Addison, J. Walker, and R. Guido, “Time-frequency analysis of biosignals," IEEE Eng Med Biol Mag, vol. 28, no. 5, pp. 14-29, Sep.-Oct. 2009.

[20] P. S. Addison and J. N. Watson, "Oxygen saturation determined using a novel wavelet ratio surface," Med Eng Phys, vol. 27, no. 3, pp. 245-8, Apr. 2005.

[21] R. W. Schafer, "What is a Savitzky-Golay filter?," IEEE Signal Processing Magazine, vol. 28, no. 4, pp. 111117, 2011.

[22] S. Palreddy, "Signal processing algorithms," in Design of Pulse Oximeter, J. G. Webster, Ed. Institute of Physics Publishing, 1997.

[23] J. M. Bland and D. G. Altman, "Statistical methods for assessing agreement between two methods of clinical measurement," Lancet, vol. 1, no. 8476, pp. 307-10, Feb. 8, 1986.

[24] S. Tungjitkusolmun, "Accuracy and errors," in Design of Pulse Oximeters, J. G. Webster, Ed. Institute of Physics Publishing, 1997. 